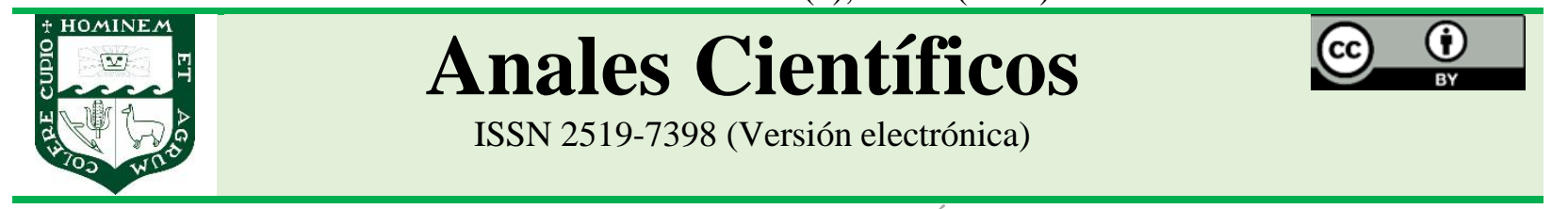

ARTÍCULO ORIGINAL - RESEARCH ARTICLE http://dx.doi.org/10.21704/ac.v82i1.1743

\title{
EL IMPACTO DE LOS CONFLICTOS LABORALES EN LAS EMPRESAS AGROEXPORTADORAS DE LIMA, DURANTE EL AÑO 2019
}

\section{The impact of labor conflicts on agro-export companies located in Lima of the year 2019}

\author{
Roberto Morales Muñoz ${ }^{1 *(1)}$ \\ ${ }^{1}$ Asociación de exportadores (ADEX), Lima, Perú. \\ *E-mail: rjmorales@lamolina.edu.pe
}

\begin{abstract}
The research describes work environment that caused labor conflicts in the agro-export companies of Lima in 2019. It also identifies the conflicts started in these companies, describes the way that conflicts are managed and the way to resolve them. The methodology used is qualitative, descriptive, cross-sectional, not experimental. The techniques used were the survey and documentary analysis, the instruments the repetitive questionnaire and digital storage. The population was made up of 811 companies and the sample 261. Four volunteers were surveyed in two stratified samples of 156 bosses and 258 collaborators. The content validity was by expert judgment and the reliability by a pilot of 10 subjects for each sample, through the Crombach Alpha and Likert scale, applying a questionnaire of 20 questions with five alternatives. The study concluded that, in 2019, the impact generated by the conflicts in the agro-export companies of the Lima region was favorable to the company and all the personnel when the conflict was properly managed. The conflicts that arose the most in these companies were identified, as well as those that appeared the least. The way that conflicts were managed in these companies was appropriate for the company and all the staff, despite the weaknesses that must be overcome. Finally, conflicts were resolved through peaceful dispute resolution mechanisms, such as negotiation, mediation, and conciliation with the support of the leadership and following up.
\end{abstract}

Keywords: agro-export companies; labor conflicts; Management; resolution

\section{RESUMEN}

La investigación describe el impacto que generaron los conflictos laborales en las empresas agroexportadoras de Lima en el año 2019; al identificar los conflictos que surgieron, la forma en que se gestionaron los conflictos y la forma de resolverlos. La metodología fue cualitativa, descriptiva, de alcance transversal, no experimental. Las técnicas fueron la encuesta y el análisis documental, los instrumentos el cuestionario repetitivo y el almacenamiento digital. La población la conformó 811 empresas y la muestra 261. Se encuestó a cuatro voluntarios en dos muestras estratificadas de 156 jefes y 258 colaboradores. La validez de contenido fue por juicio de expertos y la confiabilidad por un piloto de 10 sujetos por cada muestra, a través del Alfa de Crombach y escala Likert, mediante cuestionario de 20 preguntas con cinco alternativas. El trabajo concluyó que, el impacto que generaron los conflictos en las empresas agroexportadoras de la región Lima, en el año 2019 fue favorable a la empresa y todo el personal cuando se gestionó adecuadamente el conflicto. Se identificaron los conflictos que más surgieron en estas empresas, así 
como los que menos surgieron. La forma en que se gestionan los conflictos en estas empresas fue adecuada a la empresa y todo el personal, a pesar de que existen debilidades que se deben superar. Finalmente, los conflictos se resolvieron a través de los mecanismos pacíficos de solución de controversias, tales como la negociación, la mediación y la conciliación con el apoyo de la jefatura y dando seguimiento.

Palabras clave: empresas agroexportadoras; conflictos laborales; gestión; solución

Forma de citar el artículo (Formato APA):

Morales, R. (2021). El impacto de los conflictos laborales en las empresas agroexportadoras de Lima, durante el año 2019. Anales Científicos. 82(1), 73-82. http://dx.doi.org/10.21704/ac.v82i1.1743

Autor de correspondencia (*): Roberto Morales Muñoz. Email: rjmorales@lamolina.edu.pe

(C) Los autores. Publicado por la Universidad Nacional Agraria La Molina.

This is an open access article under the CC BY

\section{INTRODUCCIÓN}

Un conflicto es la manifestación de intereses opuestos entre dos o más partes dentro de una empresa o con externos, clientes, proveedores o el Estado, cuando se persiguen diferentes objetivos y no se puede alcanzar un acuerdo favorable. Las empresas agroexportadoras no son ajenas a estos conflictos.

Sánchez (2018) sostuvo, mientras fue ministro del sector, "El Ministerio de Trabajo y Promoción del Empleo (MTPE) solucionó 47 conflictos laborales de alcance nacional en los últimos seis meses del año, logrando beneficiar de forma directa a 81,955 trabajadores" (p.9). Asimismo, el MTPE (2020) informó que resolvieron 107 conflictos laborales y benefició a más de 195,000 trabajadores entre públicos y privados de marzo de 2018 hasta el 15 de julio de 2019.

La búsqueda de antecedentes nacionales y extranjeros permitió encontrar los trabajos de Morales (2019a) sobre conflictos en las Pequeñas y Medianas Empresas de La Molina; Morales (2019b) sobre análisis de los conflictos en las boticas y farmacias de los distritos de Lima moderna; Masías (2018) sobre conflictos laborales en Saga Falabella Bellavista; Toro (2017) y Abrego et al. (2012) sobre manejo de conflictos; Torres (2016) sobre conflictos laborales; Gascón (2013) sobre conflictos en empresas familiares, etc.

La investigación aporta una herramienta de consulta para los empresarios, los gerentes, los administradores, los colaboradores e investigadores, a quienes permitirá conocer los conflictos que surgen en estas unidades productivas, su gestión para evitarlos y enfrentarlos, así como resolverlos. El estudio se planteó el objetivo general de describir el impacto de los conflictos en las empresas agroexportadoras de Lima, en el año 2019, para ello, se plantearon tres objetivos específicos: identificar los conflictos que surgieron en las empresas, describir la forma en que se gestionaron y la forma de resolverlos.

\section{MATERIALES Y MÉTODOS}

El estudio es cualitativo, de tipo aplicado, cuyo diseño es descriptivo, no experimental de alcance transversal. La población la compuso 811 empresas agroexportadoras y la muestra fue de 261 empresas sobre la base de datos del Ministerio de la Producción del Sistema de Integrado de Información de Comercio Exterior. La fórmula utilizada fue con un nivel de confianza de $95 \%$ y un error permitido de $5 \%$. Los criterios de selección fue de voluntarios jefatura (uno) y colaboradores (tres) con un mínimo de tres años en la función y mayor de 26 años. Bajo la misma fórmula, se seleccionaron dos muestras estratificadas de jefes (156) y Colaboradores (258). El estudio utilizó tres técnicas para recoger la data: la observación, la encuesta y el análisis documental cuyos instrumentos fueron: la observación participante sistemática, el cuestionario repetitivo y el almacenamiento digital, respectivamente. $\mathrm{La}$ validez de contenido se dio por juicio de expertos cuyo resultado fue que el instrumento es aplicable y pertinente con un promedio de valoración de $88,89 \%$. La confiabilidad se realizó a través de un 
piloto de 10 sujetos de la muestra, encuestados por cada muestra estratificada, cuyo resultado, aplicando el coeficiente de confiabilidad de Alfa de Crombach, fue para los jefes de 0,994 y para los colaboradores de 0,997; altamente significativa en ambos casos para investigaciones cualitativas. Se aplicó un cuestionario de 20 preguntas con cinco alternativas de respuesta en la escala de Likert. El estudio trabajó con 40 conflictos validados por la experiencia y estudios desarrollados por Morales (2019a) que identificó los conflictos en las pequeñas y medianas empresas del distrito de La Molina y los conflictos en las boticas y farmacias en cuatro distritos de Lima moderna, Morales (2019b). Finalmente, con la información, se procedió al análisis, sistematización de datos, ordenamiento y redacción de conclusiones.

\section{RESULTADOS Y DISCUSIÓN}

Los resultados mostraron los 10 conflictos que más se presentaron en el 2019: el conflicto de liderazgo, reconocido por León (2016). Los conflictos de jerarquía reconocidos por Porret (2010) citado en Morales (2019a) y Morales (2019b). Los conflictos por falta de coordinación, reconocidos por Rosas (2017). Los conflictos inventados reconocidos por León (2016). Los conflictos de clientes insatisfechos y denunciantes reconocidos por Gascón (2013). Los conflictos por rumores, chismes y mentiras, reconocidos por Robbins \& Judge (2013) citados en De León (2018) por Gatlin et al. (2015) citados en Morales (2019a) por Pacheco y Zapata (2015), Collatupa (2018), Morales (2019a) y Morales (2019b). Los conflictos por diferencias culturales, reconocidos por Cornejo (2017), Jones y George (2014) citados en Morales (2019a) y Morales (2019b). Los conflictos por condiciones de trabajo y reconocimiento reconocidos por Rosas (2017). Los conflictos por exceso de procedimientos burocráticos reconocidos por Porret (2010) citado en Abrego et al (2010) y Morales (2019b). El conflicto imaginario es reconocido por León (2016), Morales (2019a) y Morales (2019b).

Los 10 conflictos menos frecuentes fueron los siguientes: el conflicto por evaluación de desempeño, reconocidos por León (2016) citados en
Collatupa (2018) y Morales (2019b). Los conflictos de rol reconocidos por Sánchez (2011) y Morales (2019b). Los conflictos por mal administrador, supervisor, gerente o dueño reconocidos por Morales (2019b). Los conflictos espirituales reconocidos por Jones y George (2014) citados en Morales (2019b). Los conflictos grupales reconocidos por Porret (2010) citado en Abrego et al. (2010) y Dávalos (2000) citado en Pacheco y Zapata (2015). Los conflictos de personalidad reconocidos por Newstroom (2011) citado en Collatupa (2018), Griffin y Van Fleet (2016) citados en Conejo (2017). Los conflictos personales reconocidos por León (2016), Cornejo (2017), Morales (2019b), Newstroom (2011) citado en Collatupa (2018) y Dávalos (2000) citado en Pacheco y Zapata (2015). Los conflictos de interés reconocidos por Masías (2018) y, Cavalli y Quinteros (2010) citados en Toro (2017), la Organización Internacional del Trabajo (2013) citada en Morales (2019), Granados (2007), Berroterán (2011) citado en Pujol (2015), Newstroom (2011) citado en Collatupa (2018), León (2016) y Avila (2013) citado en Rosas (2017). Los conflictos por decisiones erróneas o toma de decisiones reconocidos por Janis y Mann (1977) citados en Luna y Laca (2014) y Morales (2019b). Los conflictos por puenteo reconocidos por Morales (2019b). Los conflictos por resistencia al cambio reconocidos por Collatupa (2018) y Morales (2019b). Finalmente, los conflictos de poder sostenidos por León (2016).

Respecto al segundo objetivo, Gonzales (2006) citado en De León (2016) sienta las bases del hallazgo porque sostuvo:

La cantidad de conflictos que tenga una organización dependerá de que tan buena calidad de vida y clima haya dentro de ella y en sus relaciones de trabajo, siempre es importante buscar una solución a la dificultad que se tenga, conocer todos los detalles y agilizar la situación para tomar una decisión acertada.

En ese sentido, es necesario que las organizaciones mantengan un buen clima institucional para que eviten, enfrenten y resuelvan cualquier conflicto que pueda surgir al seno de la misma; mantener buenas 
relaciones permitirá facilitar la gestión de cualquier conflicto.

En la actualidad, muchos conflictos surgen en las organizaciones, incluso en las agroexportadoras. Sin embargo, el correcto y adecuado abordaje permite gestionarlos y resolverlos.

En ese sentido, respecto al segundo objetivo, los resultados mostraron que, la relación entre colaboradores, entre los jefes y entre colaboradores y jefes es buena, demostrando la existencia de un buen clima laboral al 97\%. Gascón (2013) planteó que, el clima organizacional es poco propicio cuando el entorno es malo y provoca tensión, los conflictos aparecen con mayor intensidad. El alto nivel de percepción entre colaboradores y jefes propician un buen entendimiento $\mathrm{y}$, por ende, una excelente satisfacción laboral en las relaciones entre ellos. Esta satisfacción está relacionada con la efectividad de los colaboradores y la jefatura para solucionar conflictos. Esta alta percepción de las buenas relaciones y el buen clima laboral, coincide y confirma el bajo nivel de propensión a los conflictos en un $92 \%$, este resultado conlleva a su alta satisfacción laboral.

De esta manera, De León y Gascón, acertadamente sentaron los pilares sobre el abordaje del conflicto, de la siguiente manera:

De León (2016) planteó:

- En una empresa muchas veces es difícil solucionar conflictos entre trabajadores o de empleados hacia jefes, por esto es importante saber cómo llevar un problema y como solucionarlo de una forma que beneficie a las partes sin crear rivalidades o dificultades mayores. Cada organización es un mundo con distintos temperamentos y a la empresa no le conviene que haya disturbios dentro de la misma.

- Ese clásico conflicto entre colaboradores y sus jefes hoy se puede gestionar, beneficiando a las partes $\mathrm{y}$, principalmente, a la empresa.

Gascón (2013) sostuvo sobre el clima organizacional o clima laboral lo siguiente:
- Clima organizacional poco propicio para el desarrollo: cuando las condiciones del entorno son malas y provocan tensión, los conflictos aparecen con mayor intensidad y frecuencia, pero cuando se trata de entornos estimulantes para que la persona pueda desarrollarse profesionalmente, esto se vuelve una ventaja.

- En efecto, un clima organizacional poco propicio incrementa la tensión y promueve la generación de conflictos con mayor intensidad. Asimismo, no menos importante es el aporte de Gonzáles (2006) sobre el clima laboral, citado en De León (2016).

- Algo que tiene relevancia en lo que es una buena organización, es el clima laboral y personal, no quiere decir que porque no surjan problemas la organización está en buenas condiciones, cuando se tiene buen clima laboral en una empresa es gracias a que los métodos que se utilizan son los adecuados para solucionar un conflicto laboral (...).

- En efecto, un buen clima laboral existe en las organizaciones porque existen métodos adecuados para solucionar los conflictos laborales. Muchas fórmulas se han escrito sobre la mejor forma de gestionar los conflictos y la forma de resolverlos, pero no se encontró ninguna acorde a las necesidades de las partes intervinientes. En ese sentido, no hay fórmulas absolutas porque cada conflicto posee su propia naturaleza, haciéndolo único.

La gestión del conflicto debe iniciar con la prevención. Sin embargo, no todas las organizaciones están preparadas para prevenirlo porque lo consideran innecesario. Asimismo, si el conflicto ya surgió se debe enfrentar hasta resolverlo.

La gestión de conflictos sirve para prevenir y encontrar las claves que los solucionen. En relación a los motivos que generan los conflictos, los resultados demuestran que los conflictos son ocasionados por motivos personales $47 \%$, sostenido por (Robbins y Judge, 2013; López, 2013) citados en 
Gascón (2013). Asimismo, el síndrome de Bourout $31 \%$, reconocido por Vidal (2018), Conejo (2017), Prieto (2012), así como (Brill, 1984; Schaufeli y Buunk, 2003), citados en Prieto (2012). La falta de liderazgo 29\% reconocido por Yarce (2013) citado en De León (2016). La falta de protocolos, guías, manuales, normas o procedimientos sobre el manejo de conflicto $26 \%$ reconocido por López (2013) citado en De León (2016), Pacheco y Zapata (2015), Valdés (1997). La falta de capacitación, de coaching o inducción $26 \%$ y $23 \%$ reconocido por Gascón (2013), Gálvez (2014) citado por De León (2016) y De León (2018). La angustia, ansiedad y otros $14 \%$ reconocida por Abrego et al (2013), Bolger, et al. (1989) citado en Prieto (2012) y Prieto (2012). El mobbing $8 \%$ reconocido por Vidal (2018) y la falta de inteligencia emocional 4\% reconocida por Gálvez (2014) citado en De León (2016) y Abrego et al. (2013).

Ya Cornejo (2018) lo había afirmado en su estudio: Los directivos de la Municipalidad Distrital de Sayán con la finalidad de solucionar los problemas deben tomar algunas medidas como el área encargada de los recursos humanos se encargue de realizar capacitaciones para fomentar y fortalecer el uso de estrategias de negociación para la solución de conflictos en sus áreas donde laboran.

En efecto, es de vital importancia que la empresa capacite al personal sobre manejo de conflictos, a fin de evitarlos y resolverlos.

Por otro lado, sobre la existencia de guías y manuales concuerda en un $33 \%$, los resultados demuestran que sí existen. Sin embargo, el $61 \%$ considera que no existen y solo un $6 \%$ se muestra indiferente. La falta de protocolos, guías, manuales, normas o procedimientos sobre el manejo de conflicto, son negativos para la solución de conflictos, reconocido por López (2013), citado en De León (2016) y por Pacheco y Zapata (2015), Valdés (1997), citado en Abrego et al. (2013) y León (2016).

Asimismo, hubo coincidencia en la existencia de capacitación, coaching o inducción sobre manejo de conflictos en $89 \%$, el $10 \%$ considera que no existe y el 1\% fue indiferente. León (2016) planteó que las jefaturas deben recibir algún tipo de formación de gestión de conflictos.

Respecto a las consecuencias, los resultados demuestran que hay consecuencias constructivas en los conflictos con una coincidencia del $91 \%$, el $3 \%$ no coincide y un $6 \%$ fue indiferente. Así, Coser y Simmel citados por León (2016) plantearon la existencia de aspectos positivos del conflicto.

Respecto al tercer objetivo, se obtuvo que los conflictos fueron resueltos en un $79 \%$ de coincidencia, solo el $6 \%$ no coincidió y el $15 \%$ se mostró indiferente. Asimismo, sobre la intervención de la jefatura, hubo coincidencia de intervención en $64 \%$, el $12 \%$ no coincidió y un $24 \%$ se mostró indiferente, siendo alto en ambos casos. Escudero (2011) citado en De León (2016) definió los conflictos "los conflictos laborales como el origen de puntos de vista de dos personas totalmente diferentes, que surgen en el ámbito y forman tensiones que saltan a la superficie". En la mayoría de los casos, el problema es pequeño en realidad. Por ello, más adelante sostiene que alguna de las partes tiende a evitar detalles que surgieron y la persona que habitualmente es el mediador en estas situaciones es el jefe, ya que conoce ciertas causas, temperamentos, relaciones y formas de ser de los empleados; entre más rápido se detecte un conflicto dentro de una organización puede ser más factible encontrarle una solución al tratar el problema, ya que entre más pasa el tiempo la dificultad será más complicada, se pueden descubrir ciertas diferencias entre colaboradores con preguntas y actividades. En ese sentido, desde el inicio del problema hay que abordarlo y solucionarlo, antes que desencadene en un conflicto, que genere daños a la empresa y a todo el personal.

Masías (2018), recomendó que el jefe de área debe detectar los conflictos a tiempo para evitarlos. De León (2016) en su estudio afirmó, lo expuesto por los encuestados: "Manifiestan que al momento de surgir algún conflicto se dirigen principalmente al jefe inmediato". Más adelante planteó: "El 82\% de los colaboradores manifestó que recurren a su jefe inmediato en caso de que haya conflictos dentro de su área de trabajo". 
León (2016) en su trabajo sostuvo que el conflicto, en el lugar de trabajo, puede tener diferentes efectos en función de cómo se gestione.

Un buen directivo o jefe puede y debe identificar conflictos positivos y fomentar ese tipo de interacción entre los empleados, siempre y cuando como exponía anteriormente la empresa le haya dado la formación en dicho sentido. Los jefes deben identificar un conflicto y resolverlo inmediatamente, como sea posible.

La jefatura interviene en todo momento y saca lo mejor de cada situación porque conoce a los colaboradores, según el autor. Masías (2018) planteó respecto a la problemática de Saga Falabella de Bellavista, especialmente en el área de ventas:

Se generan conflictos laborales que están afectando el rendimiento del trabajador, así como el servicio que se ofrece a los clientes. Para ello, se recomienda que el jefe de área debe detectar estos conflictos a tiempo para evitar los resultados negativos en las evaluaciones realizadas por el departamento de evaluación y desarrollo del personal.

Cuando se agotó la prevención es necesario enfrentar el conflicto y solucionarlo. De León (2014) sostuvo sobre el manejo del conflicto, lo siguiente: El mal manejo que se tenga un conflicto puede empeorarlo, pero si al tomar alguna decisión se estudia minuciosamente el caso donde las dos partes salgan beneficiadas se puede reforzar y hacer crecer la organización, al momento que la calidad de lo que se decide en la empresa va de mejor manera, también repercute en los demás porque se fomentan las relaciones, comunicación y la creatividad, de esta manera es beneficioso un percance laboral.

Hoy, para enfrentar y resolver un conflicto se debe utilizar la creatividad, la objetividad, la imparcialidad y el análisis crítico para estimular el pensamiento de las partes enfrentadas, llamar a la razón, al criterio común y la buena fe, a fin de resolver pacíficamente, en función a los intereses. De León (2018) también se refirió a la creatividad y asertividad para gestionar el conflicto.
Lo ideal, como ya se sostuvo, es lograr que el conflicto sea productivo, cambiar los efectos negativos en positivos en beneficio de todo el personal, aspectos laborales y organizacionales. Este procedimiento coincide con lo planteado por Toro (2017) sobre la gestión a desarrollar para enfrentar el conflicto.

Se puede apreciar que, De León (2017) y Toro (2017) coincidieron plenamente en las etapas para enfrentar los conflictos. Pujol (2015) siguiendo a Lozano (2011) afirmó que cada persona enfrenta el conflicto de manera diferente, describe diferentes estilos de gestión de conflictos basándose en Alzate (2015) y De Diego y Guillén (2008) los cuales se refirieron a la negación, la evitación, la acomodación, el enfrentamiento, la colaboración y el compromiso. Sin embargo, el último, plantea una diferencia porque los tres primeros estilos no solucionan, sino huyen de ella, la evitan pudiendo elevar el grado de efervescencia de este o elevar su complejidad. Incluso, la OIT (2013) planteó su enfoque, como el poder (cuando una parte emplea la coacción para forzar a la otra), los derechos (cuando se usa la norma de derecho o equidad para resolverlo) y el consenso (cuando se empeñan en conciliar, transigir o adoptar posturas o necesidades para resolverlo). Esto permite un posicionamiento estratégico para tomar decisiones y el mejor manejo que se le puede dar, citado en Morales (2019a).

Mendías (2002) planteó un correcto abordaje, tratamiento y solución de los conflictos, descartando la conducta elusiva (eludir, ignorar, no abordar) y la adversa (enfrentar legalmente a la otra parte). Sin embargo, la conducta negociadora (encarar las partes el problema y solucionarla), plantea muchas ventajas (ahorro de tiempo y dinero, mejora la relación, etc.).

Mendías (2002) realizó un interesante aporte sobre el abordaje, tratamiento y solución de los conflictos a través de diversos caminos, con una conducta elusiva (eludir, ignorar, no abordar), sin ventaja y muchas desventajas (el incremento del conflicto, por ejemplo).

Finalmente, la gestión de conflictos implica la obligatoria disposición de una guía, manual, 
protocolo o plan de acción para evitar, enfrentar y resolver los conflictos. La fortaleza del protocolo radica en elaborarlo correctamente y que el personal lo aplique o cumpla. En efecto, el buen manejo, la buena gestión del conflicto permite que todas las partes ganen, el clima laboral y las relaciones entre todo el personal, entre otros.

Respecto al tercer objetivo, a la variopinta diversidad bibliográfica, tanto nacional e internacional, existen grandes coincidencias dentro del marco de lo clásico o tradicional. Se indagó sobre siete técnicas específicas para resolver los conflictos empresariales, tales como facilitación, mediación, conciliación, indagación o decisión de expertos, negociación, arbitraje y el poder judicial En efecto, las formas de resolver los conflictos son diversas. El correcto y adecuado abordaje permite la solución porque toda situación es diferente y merece ser tratada desde su propia naturaleza. Muchas fórmulas han tratado de dar respuesta, pero no se encontró ninguna acorde a las necesidades de las partes intervinientes.

- Gonzales (2006) citado en De León (2016) reconoció "la imposición, la conciliación", "la negociación, la negociación asistida, el arbitraje (voluntario e impuesto)"; "la ley, el arbitraje y la mediación"; también citado por Abrego et al. (2013) quienes se adscribieron a lo planteado por Gonzáles sobre los métodos: "Negociación (Sinibaldi, 2009) y Conciliación (Martínez, 1995)"; Mediación (Jaramillo, 1996), Arbitraje (Jaramillo, 1996), Solución Jurisdiccional (González, 2006), Buenos Oficios (González, 2006), y Decisión Administrativa (González, 2006)". En este extremo, se agregan los buenos oficios y la decisión administrativa, adicionales a los cinco métodos tradicionales de solución pacífica de controversias.

- De León (2018) reconoció "la mediación".

- De la Cruz (2014) citado en De León (2016) comentó que "la negociación es una de las técnicas que se utilizan para la solución de conflictos laborales".

- Gascón (2013) reconoció a diversas personas según su accionar: "Un interventor efectivo, ya sea facilitador, mediador, árbitro o manipulador de reglas, debe entender el proceso de negociación desde diferentes ángulos" . Incluso, reconoció la "negociación" y dedicó un espacio amplio "negociación como elemento de solución de conflictos".

- León (2016) reconoció "los procesos de negociación, mediación, conciliación o arbitraje". Asimismo, para la resolución de conflictos (Thomas, 1976; Rahim y Bonoma, 1979; Vera (2004) citados en Masías (2018) resaltaron "la negociación, la mediación, el arbitraje y el juicio".

- Robbins (2011) citado en Pacheco y Zapata (2015) planteó los métodos más utilizados, según lo investigado: “a) negociación, b) arbitraje, c) mediación, d) conciliación, e) amigable composición".

- Prieto (2012) reconoció como método de solución de conflictos "la negociación (Ertel, 2008; Chiavenato, 2007), la mediación (Kressel y Pruitt, 1985)". "El arbitraje y el juicio". Por su parte, Sebastián (2017) planteó como principales estrategias: “a) la negociación (MINEDU, 2013 y Robbins, 2004)”. "b) La mediación, c) la conciliación y d) el arbitraje (Robbins, 2004)".

- Torres (2016) reconoció: "la negociación, la mediación, el arbitraje y el juicio".

En referencia al tercer objetivo, los resultados demostraron que el medio utilizado para resolver los conflictos fueron: la negociación en $56 \%$ reconocido por De la Cruz (2014) citado en De León (2016), Robbins y Judge (2013) citados en Pacheco y Zapata (2011), Gonzales (2006) citado en De León (2016) y Abrego et al. (2013), Conejo (2018), Gascón (2013), León (2016), (Thomas, 1976; Rahim y Bonoma,1979; Vera, 2004) citados en Masías (2018), Robbins (2011) citado en Pacheco y Zapata (2015), (Coleman, 2006; Crampton, 2008; Swenson, 1999; Chiavenato, 2007; Ertel, 2008) citados en Prieto (2012), (MINEDU, 2013; Robbins, 2004), citados en Sebastián (2017) y Torres (2016). La mediación en 23\%, Robbins y Judge (2013) citados en Pacheco y Zapata (2011), Gonzales (2006) citado en De León (2016) y Abrego et al. (2013), De León (2018), Gascón (2013), León (2016), (Thomas, 1976; Rahim y Bonoma,1979; Vera, 2004) citados en Masías (2018), Robbins (2011) citado en Pacheco y Zapata (2015), (Kressel y Pruitt, 1985; (Coleman, 2006; Crampton, 2008; Swenson, 1999) citados en 
Prieto (2012), Sebastián (2017) y Torres (2016). La conciliación en 20\% Robbins y Judge (2013) citados en Pacheco y Zapata (2011), Gonzales (2006) citado en De León (2016) y Abrego et al. (2013), León (2016), Robbins (2011) citado en Pacheco y Zapata (2015), (Coleman, 2006; Crampton, 2008; Swenson, 1999) citados en Prieto (2012) y Sebastián (2017). El poder judicial fue el 1\%, Gonzales (2006) citado en De León (2016) y Abrego et al (2013), (Thomas, 1976; Rahim y Bonoma,1979; Vera, 2004) citados en Masías (2018), (Coleman, 2006; Crampton, 2008; Swenson, 1999) citados en Prieto (2012) y Torres (2016). El arbitraje no fue considerado.

Asimismo, los resultados mostraron que, hubo una fuerte coincidencia en que el medio más adecuado para resolver un conflicto es la negociación en $76 \%$, la mediación en $23 \%$ y la conciliación en $1 \%$. Asimismo, hubo seguimiento a los conflictos en $35 \%$, un $44 \%$ coincide que no hubo seguimiento y un $21 \%$ mostró indiferencia. Este rechazo es importante, peor aún la cantidad de indiferentes. Merlano (2011) citado en De León (2018) sostuvo que será necesario darle seguimiento a la estrategia y aplicar talleres de trabajo en equipo que mejoren la salud de los colaboradores.

Sobre las consecuencias negativas generadas por los conflictos, los resultados muestran que las coincidencias son las siguientes: una gestión deficiente de la jefatura en 30\%; carencia de guías, manuales, coaching, capacitación en conflictos en $22 \%$; carencia de políticas públicas en $20 \%$; carencia de destrezas, habilidades y competencias de los involucrados en 16\%); y carencia de visión positiva de los involucrados (sentimientos $\mathrm{y}$ emociones) en $12 \%$.

Al encontrar la solución, sea la que sea, siempre se le debe dar seguimiento. Merlano (2011) citado De León (2018) sostuvo que: "para resolver un conflicto es necesario analizar su origen y naturaleza, luego extraer la información necesaria para formar líneas abiertas de discusión (...)". En su estudio, preguntó si es fácil que se origine un malentendido, 28 trabajadores respondieron que sí..." por lo tanto únicamente será necesario darle seguimiento a la estrategia, además de aplicar talleres de trabajo en equipo, relaciones interpersonales y talleres que mejoren la salud emocional de los colaboradores".

\section{CONCLUSIONES}

El impacto que generaron los conflictos en las empresas agroexportadoras de la región Lima, en el año 2019 fue favorable a la empresa y todo el personal cuando se gestionó adecuadamente el conflicto. Se cumplió el primer objetivo al identificar los conflictos que más surgieron $\mathrm{n}$ en las empresas agroexportadoras de la región Lima, así como los que menos surgieron, los cuales son diferentes a los que surgen en las Pequeñas y Medianas Empresas del distrito de La Molina y las Pequeñas Empresas del sector boticas y farmacias de los distritos de Lima moderna.

La forma en que se gestionaron los conflictos en las empresas agroexportadoras fue favorable a la empresa y todo el personal, a pesar de que existen debilidades que se deben fortalecer; por ejemplo, capacitar al personal sobre manejo de conflictos, coaching, sobre todo a la Jefatura; elaborar manuales, guías o protocolos de procedimientos que permitan evitar, enfrentar y resolver los conflictos, en cualquiera de sus etapas, modalidades y naturaleza. Se demostró la buena gestión a través de la buena relación entre colaboradores y jefaturas, la baja frecuencia con que surgen los conflictos y que no hay reducción de la productividad o desempeño. Asimismo, no hay consecuencias negativas por la existencia de un buen clima laboral y un ambiente positivo, incluso, luego de un conflicto, cumpliéndose el segundo objetivo.

Finalmente, sobre el tercer objetivo, la forma en que se resolvieron los conflictos en las empresas agroexportadoras fue a través de los mecanismos pacíficos de solución de controversias, tales como la negociación, la mediación y la conciliación con el apoyo de la jefatura y dando seguimiento para evitar cualquier resurgimiento.

Se pueden generar nuevas investigaciones sobre el efecto de los conflictos en la productividad y desempeño de los colaboradores y jefatura, el 
impacto positivo o negativo de los conflictos en las empresas agroexportadoras o el efecto que causan los conflictos en la salud del personal, así como en los ámbitos laboral y empresarial.

\section{Conflictos de intereses}

Los autores firmantes del presente trabajo de investigación declaran no tener ningún potencial conflicto de interés personal o económico con otras personas $\mathrm{u}$ organizaciones que puedan influir indebidamente con el presente manuscrito.

\section{Contribuciones de los autores}

Preparación y ejecución: RM; Desarrollo de la metodología: RM; Concepción y diseño: RM; Edición del artículo: RM; Supervisión del estudio: RM.

\section{LITERATURA CITADA}

- Abrego, R., González, K., \& Lullo, R. (2012). Elaboración de un diseño instruccional para el desarrollo de habilidades en el manejo de conflictos y negociaciones dirigidas al área de mercadeo y ventas en la industria farmacéutica Roka C.A. utilizando la inteligencia emocional. Tesis Lic. Caracas, Venezuela, Universidad Central de Venezuela. 144 p.

- Collatupa, L. (2018). Efectos de los conflictos interpersonales en el desempeño laboral de la dirección regional de educación Puno 2017 - I. Tesis Lic. Universidad Nacional del Altiplano. $95 \mathrm{p}$.

- Conejo, A. (2017). Manejo de conflictos y su influencia en el estrés laboral del personal administrativo de la Municipalidad distrital de Sayán, 2017. Tesis Mg. Huacho, Lima, Universidad Nacional José Faustino Sánchez Carrión. 74 p.

- De León, BL. (2018). Comunicación interna y manejo de conflictos. Tesis Lic. Quetzaltenango, Guatemala, Universidad Rafael Landívar.74 p.

- De León, M. (2016). Liderazgo y conflictos laborales. Tesis Lic. Quetzaltenango, Guatemala, Universidad Rafael Landívar. 88 p.

- Gascón, S. (2013). Conflictos en empresas familiares. Tesis Lic. Mendoza, Argentina, Universidad Nacional de Cuyo. 96 p.
- Masías, J. (2018). Los conflictos laborales y el servicio de excelencia de los empleados de la empresa Saga Falabella Bellavista, 2018. Tesis Mg. Lima, Perú, Universidad Cesar Vallejo. 72 p.

- Mendías, N. (2002). Solución de conflictos empresariales. Editorial Carpetas de Derecho. Buenos Aires, Argentina. 253 p.

- MTPE [Ministerio de Trabajo y Promoción del Empleo, Perú] (2020). MTPE resolvió 107 conflictos laborales y benefició a más de 195,000 trabajadores. Agencia Andina. Recuperado de https://andina.pe/agencia/noticia-mtpe-resolvio107-conflictos-laborales-y-beneficio-a-mas195000-trabajadores-760506.aspx

- Morales, R. $\left(2019^{\mathrm{a}}\right)$. Los conflictos empresariales en las boticas y farmacias en Lima moderna. Revista Anales Científicos, 80(2), 421436.

- Morales, R. (2019b). Los conflictos empresariales en las boticas y farmacias en Lima moderna. Revista Anales Científicos, 80(1), 2639.

- Pacheco, M., \& Zapata, J. (2015). Técnicas de resolución de conflictos en el área laboral de la empresa Procongelados S.A, ubicada en la Provincia de Pichincha, Cantón Mejía Parroquia Aloag, Barrio San Alfonso en el Periodo 20132014. Tesis Lic. Latacunga, Ecuador, Universidad Técnica de Cotopaxi. 113 p.

- Prieto, J. (2012). Calidad de vida laboral de negociadores y mediadores de conflictos de España y Paraguay. Tesis Dr. Salamanca, Reino de España, Universidad de Salamanca. 308 p.

- Pujol, MJ. (2015). Gestión y Resolución de conflictos: La mediación. Tesis Lic. Islas Baleares, Reino de España, Universitat de le Illes Balears. 35 p.

- Rosas, E. (2017). Conflicto y desempeño laboral en la SBP Huaraz 2017. Tesis Lic. Ancash, Perú, Universidad San Pedro. 96 p.

- Sánchez, C. (2018). MTPE resuelve 47 conflictos laborales en últimos seis meses y beneficia a casi 82 mil trabajadores. Diario Gestión, p.09.

- Sánchez, M.E., Cegarra, D., \& Cegarra J. (2011). ¿Influye el conflicto trabajo-vida personal de los 
empleados en la empresa?, Universia Business Review, Fundación Universia, 29, 100-115.

- Sebastián, G. (2017). La inteligencia emocional y el estilo de manejo de conflictos de los docentes en las instituciones educativas públicas de la RED 9, UGEL 06, Vitarte, Lima. Tesis Mg. Lima, Perú. Universidad Nacional de Educación. $154 \mathrm{p}$.

- Toro, C.C. (2017). Propuesta para el manejo de conflictos en el área de mantenimiento de la
Universidad Central del Ecuador. Tesis Mg. Quito, Ecuador, Universidad Central del Ecuador. 133 p.

- Torres, Y. (2016). Conflicto laboral y desempeño de los trabajadores de las MYPES familiares del sector calzado del distrito El Porvenir 2016. Tesis Lic. Trujillo, Perú, Univ. César Vallejo. 82 